\title{
COMORBIDADE PSIQUIÁTRICA DIMINUI A QUALIDADE DE VIDA DE PACIENTES COM ENXAQUECA CRÔNICA
}

\author{
Juliane Prieto Peres Mercante ${ }^{1,2}$, Marcio Antonini Bernik ${ }^{1,2}$, \\ Vera Zukerman-Guendler ${ }^{1,3}$, Eliova Zukerman', \\ Evelyn Kuczynski', Mario Fernando Prieto Peres ${ }^{1,3}$
}

\begin{abstract}
RESUMO - Introdução: A enxaqueca apresenta elevada comorbidade com os transtornos de humor e de ansiedade e extremo impacto no grau de incapacidade e qualidade de vida do indivíduo afetado, mas pouco se sabe sobre a qualidade de vida dos pacientes com enxaqueca crônica e o impacto das comorbidades psiquiátricas. Método: Cinquenta pacientes com diagnóstico de enxaqueca crônica (Sociedade Internacional de Cefaléias, 2004) foram diagnosticados quanto à presença de transtornos mentais, através da entrevista estruturada SCID-I/P e do questionário de qualidade de vida SF-36. Pacientes foram divididos nos seguintes grupos: enxaqueca crônica com transtornos de ansiedade, transtorno de ansiedade generalizada, transtornos de ansiedade com pelo menos um episódio depressivo maior, com episódio depressivo maior, comparados a pacientes sem comorbidade psiquiátrica. Resultados: Constatou-se qualidade de vida significativamente inferior nos oito domínios da SF-36, nos pacientes com enxaqueca crônica associada a comorbidade psiquiátrica tais como transtornos de ansiedade, transtorno de ansiedade generalizada, transtornos de ansiedade com pelo menos um episódio depressivo maior, assim como nos pacientes apenas com episódio depressivo maior, comparados a pacientes com enxaqueca crônica sem comorbidade psiquiátrica $(p<0,05)$. Somente no domínio Estado Geral de Saúde do SF-36, a qualidade de vida não foi significativamente inferior em todos os grupos de pacientes com enxaqueca crônica associada a comorbidade psiquiátrica. Nos domínios do SF-36 relacionados aos aspectos físicos, a qualidade de vida não foi significativamente inferior somente nos transtornos de ansiedade. Conclusão: A comorbidade da enxaqueca crônica e transtornos mentais é fator associado à piora da qualidade de vida dos pacientes e deve ser ativamente pesquisada nesta população.
\end{abstract}

PALAVRAS-CHAVE: enxaqueca crônica, comorbidade psiquiátrica, qualidade de vida.

\section{Psychiatric comorbidities decrease quality of life in chronic migraine patients}

\begin{abstract}
Introduction: Chronic migraine is a common, debilitating condition affecting quality of life and social functioning with significant impact. Migraine is highly comorbid with anxiety and mood disorders, but little is known about psychiatric comorbidities impact in the migraine patient quality of life. Method: Fifty patients with chronic migraine diagnosed according to the International Headache Society (2004) were interviewed and met diagnostic criteria for mental disorders, according to the structured interview SCIDI/P and were evaluated by the SF-36 Health Survey questionnaire. Patients were divided in the following groups: chronic migraine with both mood and anxiety disorders, with only anxiety disorders, with generalized anxiety disorder, with only a mood disorder, and without psychopathology. The scores in the group without psychopathology were compared with the other groups. All eight domains of the SF-36 scale were compared in those groups. Results: Significantly lower $(p<0.05)$ quality of life was found on all eight SF36 domains for CM psychiatric comorbidity patients compared to no-co morbidity patients. On the SF-36 General Health domain alone, quality of life was not significantly lower for all four CM psychiatric comorbidity groups. On the SF-36 Physical Aspects domain alone, quality of life was not significantly lower only for the Anxiety Disorders group. Conclusion: Chronic migraine comorbidity with mental disorder is a significant factor affecting patients' quality of life.
\end{abstract}

KEY WORDS: chronic migraine, psychiatric comorbidities, quality of life.

\footnotetext{
${ }^{1}$ Instituto de Ensino e Pesquisa. Hospital Israelita Albert Einstein, São Paulo SP, Brasil; ${ }^{2}$ Instituto de Psiquiatria, Hospital das Clínicas da FMUSP, São Paulo SP, Brasil; ${ }^{3}$ UNIFESP, Escola Paulista de Medicina, São Paulo SP, Brasil.
}

Recebido 16 Janeiro 2007, recebido na forma final 8 Maio 2007. Aceito 4 Junho 2007. 
A avaliação de qualidade de vida tem sido cada vez mais utilizada na área da saúde, principalmente depois que suas propriedades de medida foram comprovadas como um parâmetro válido e reprodutível. Medir o impacto de determinada doença na qualidade de vida do paciente tem se tornado cada vez mais importante 1 . De acordo com a segunda e revisada edição da Classificação da Sociedade Internacional das Cefaléias de 2004², a enxaqueca ou migrânea crônica foi recentemente redefinida pela ocorrência de crises com freqüência igual ou maior que 8 dias por mês (anteriormente 15) por mais de três meses, na ausência de uso excessivo de medicação, com uma prevalência de 2 a $3 \%$ da população geral ${ }^{3-5}$. É também comum nos centros especializados em cefaléias, onde representa até $60 \%$ das consultas, além de serem indivíduos de manejo mais complexo e pior resposta a tratamento ${ }^{6}$. É uma condição médica de extremo impacto na sociedade e no indivíduo afetado ${ }^{7}$. $\mathrm{Na}$ organização mundial de saúde, ocupa a $19^{\mathrm{a}}$ posição mundial entre todas as doenças causadoras de incapacidade. Os transtornos mentais estão associados a um intenso grau de incapacidade, baixo nível de satisfação e qualidade de vida, em parte pela elevada comorbidade com outros problemas médicos ${ }^{8}$.

A informação epidemiológica mais consistente até o momento, o Epidemiologic Catchment Área9, mostrou que os transtornos de ansiedade como um todo, é o grupo de transtornos mentais mais prevalentes na população. Um estudo posterior, o National Comorbidity Survey confirmou uma prevalência de transtornos de ansiedade ao longo da vida de $24 \%{ }^{10}$. No Brasil, um estudo multicêntrico sobre epidemiologia de transtornos mentais realizado em três grandes cidades (São Paulo, Brasília e Porto Alegre) também mostrou que os transtornos de ansiedade apresentam a prevalência mais elevada, acima de $18 \%{ }^{11}$.

Vários levantamentos mostram que portadores de enxaqueca crônica apresentam elevada comorbidade com transtornos de humor e transtornos de ansiedade $^{12-17}$. Alguns estudos têm mostrado que portadores de enxaqueca têm significativas limitações na qualidade de vida em relação à população saudável e também quando comparadas a outras condições crônicas. $O$ questionário genérico mais usado para enxaqueca é o Medical Outcome Survey 36 Item ShortForm Study, SF-36 ${ }^{18}$. Apesar das grandes implicações das comorbidades psiquiátricas no manejo da enxaqueca, pouco se sabe sobre o seu impacto na qualidade de vida dos pacientes. O objetivo deste estudo foi avaliar o possível impacto das comorbidades psiquiátricas na qualidade de vida de pacientes com enxaqueca crônica.

\section{MÉTODO}

Foram estudados 50 pacientes oriundos de uma clínica de cefaléia terciária (Hospital Albert Einstein) sem tratamento psiquiátrico ou uso de psicofármacos atual. Todos preencheram os critérios de diagnóstico de enxaqueca crônica (ou provável enxaqueca crônica), de acordo com a segunda e revisada edição da classificação da Sociedade Internacional de Cefaléias (2004) e recentemente redefinida ${ }^{2}$ em um conceito que inclui a cefaléia da migrânea ocorrendo em 8 (anteriormente 15) ou mais dias por mês por mais de três meses, na ausência de uso excessivo de medicação.

Todos os pacientes foram submetidos à entrevista estruturada SCID-I/P, que utiliza os critérios diagnósticos do DSM-IV para transtornos mentais ${ }^{19}$ e ao questionário genérico para avaliar qualidade de vida: Medical Outcome Study 36 Item Short-form Health Survey SF- $36^{18}$, que abrange oito aspectos da qualidade de vida: capacidade funcional, aspectos físicos, aspectos emocionais, dor, estado geral de saúde, vitalidade, aspectos sociais e saúde mental. Pacientes foram divididos nos seguintes grupos: enxaqueca crônica associada a transtornos de ansiedade, transtorno de ansiedade generalizada, transtornos de ansiedade com pelo menos um episódio depressivo maior, com episódio depressivo maior e comparados a pacientes com enxaqueca crônica sem comorbidade psiquiátrica. Os oito domínios do questionário SF-36 foram analisados. O estudo foi aprovado pelo Comitê de Ética em Pesquisa e os pacientes assinaram consentimento informado.

A análise estatística foi realizada comparando-se os valores dos escores do questionário SF-36 em pacientes com e sem comorbidades psiquiátricas. O teste t de Student foi utilizado para comparar as amostras pareadas, após averiguação de distribuição normal. Caso necessário, o teste Mann-Whitney também foi utilizado. $\mathrm{O} p$ foi considerado estatisticamente significante se menor que $<0,05$.

\section{RESULTADOS}

Quarenta e dois pacientes (84\%) preencheram critério diagnóstico ao longo da vida para um transtorno mental. Trinta e oito (76\%) apresentaram algum transtorno de ansiedade; 25 (50\%) apresentaram algum transtorno do humor; e 22 (44\%) apresentaram um transtorno de ansiedade e um transtorno do humor. Vinte e seis (52\%) apresentaram diagnóstico de transtorno de ansiedade generalizada (TAG); 3 (6\%) apresentaram diagnóstico de transtorno de pânico; 2 (4\%) transtorno obsessivo-compulsivo; três (6\%), de transtorno de estresse pós-traumático, e 27 (54\%), fobia, social ou específica. Vinte e dois (44\%) apresentaram episódio depressivo maior, sendo 14 (28\%) atuais. Onze (22\%) com um episódio único e onze (22\%), com episódios recorrentes. Dois pacientes tiveram diagnóstico de distimia e dois de transtorno bipolar. A Tabela 1 mostra os resultados médios quanto às escalas do SF-36. Observa-se qualidade de vida significativamente inferior nos oito domínios da escala SF-36, nos pacientes com enxaqueca crônica asso- 
Tabela 1. Resultados médios das escalas do SF-36 nos pacientes com enxaqueca crônica com e sem comorbidades psiquiátricas.

\begin{tabular}{lccccc}
\hline & $\begin{array}{c}\text { Sem comorbidade } \\
\text { psiquiátrica }\end{array}$ & $\begin{array}{c}\text { Transtornos } \\
\text { de ansiedade }\end{array}$ & $\begin{array}{c}\text { Transtorno de } \\
\text { ansiedade } \\
\text { generalizada }\end{array}$ & $\begin{array}{c}\text { Transtornos de } \\
\text { ansiedade }+ \\
\text { episódio } \\
\text { depressivo maior }\end{array}$ & $\begin{array}{c}\text { Episódio } \\
\text { depressivo maior }\end{array}$ \\
\hline Capacidade funcional & 74,37 & 60,12 & 59,03 & 50,83 & 52,95 \\
Aspectos físicos & 53,12 & 43,90 & 38,46 & 23,61 & 20,45 \\
Dor & 37,75 & 29,68 & 27,84 & 26 & 26,63 \\
Estado geral de saúde & 65,87 & 59,65 & 63,57 & 57,55 & 59,63 \\
Vitalidade & 55 & 39,63 & 37,5 & 32,22 & 31,81 \\
Aspectos sociais & 65,62 & 54,26 & 48,55 & 48,61 & 48,86 \\
Aspecto emocional & 79,16 & 56,91 & 50 & 51,85 & 36 \\
Saúde mental & 73 & 45,36 & 41,38 & 36,81 & 30 \\
\hline
\end{tabular}

Tabela 2. Componentes do SF-36 nos pacientes com enxaqueca crônica com e sem comorbidades psiquiátricas.

\begin{tabular}{|c|c|c|c|c|c|c|c|c|}
\hline $\begin{array}{c}\text { SCID } \\
0 \text { versus }\end{array}$ & $\begin{array}{l}\text { Capacida- } \\
\text { de funcional }\end{array}$ & $\begin{array}{l}\text { Aspectos } \\
\text { físicos }\end{array}$ & Dor & $\begin{array}{l}\text { Estado geral } \\
\text { de saúde }\end{array}$ & Vitalidade & $\begin{array}{l}\text { Aspectos } \\
\text { sociais }\end{array}$ & $\begin{array}{l}\text { Aspecto } \\
\text { emocional }\end{array}$ & $\begin{array}{l}\text { Saúde } \\
\text { mental }\end{array}$ \\
\hline TAG+EDM & 0,02 & 0,08 & 0,02 & 0,22 & 0,006 & 0,003 & 0,01 & $<0,001$ \\
\hline TAG & 0,04 & 0,17 & 0,05 & 0,39 & 0,01 & 0,004 & 0,01 & $<0,001$ \\
\hline EDM & 0,02 & 0,01 & 0,02 & 0,23 & 0,002 & 0,016 & 0,01 & $<0,001$ \\
\hline
\end{tabular}

TAG, transtorno de ansiedade generalizada; EDM, episódio depressivo.

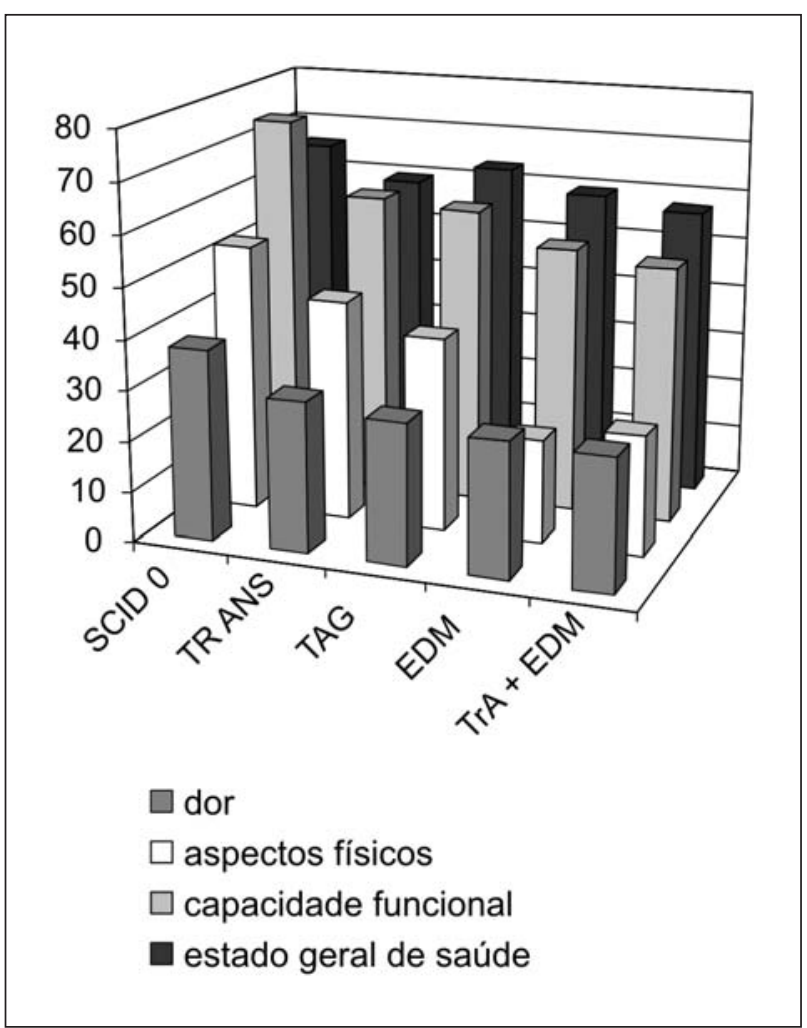

Fig 1. Distribuição dos escores da escala SF-36 nos subitens: dor, aspectos físicos, capacidade funcional e estado geral de saúde, em pacientes migranosos sem comorbidade psiquiátrica (SCID 0), com algum transtorno de ansiedade (Tr ANS), com transtorno de ansiedade generalizada (TAG), com algum episódio depressivo maior (EDM), e com transtorno de ansiedade mais episódio depressivo maior ( $\operatorname{Tr} A+E D M)$.

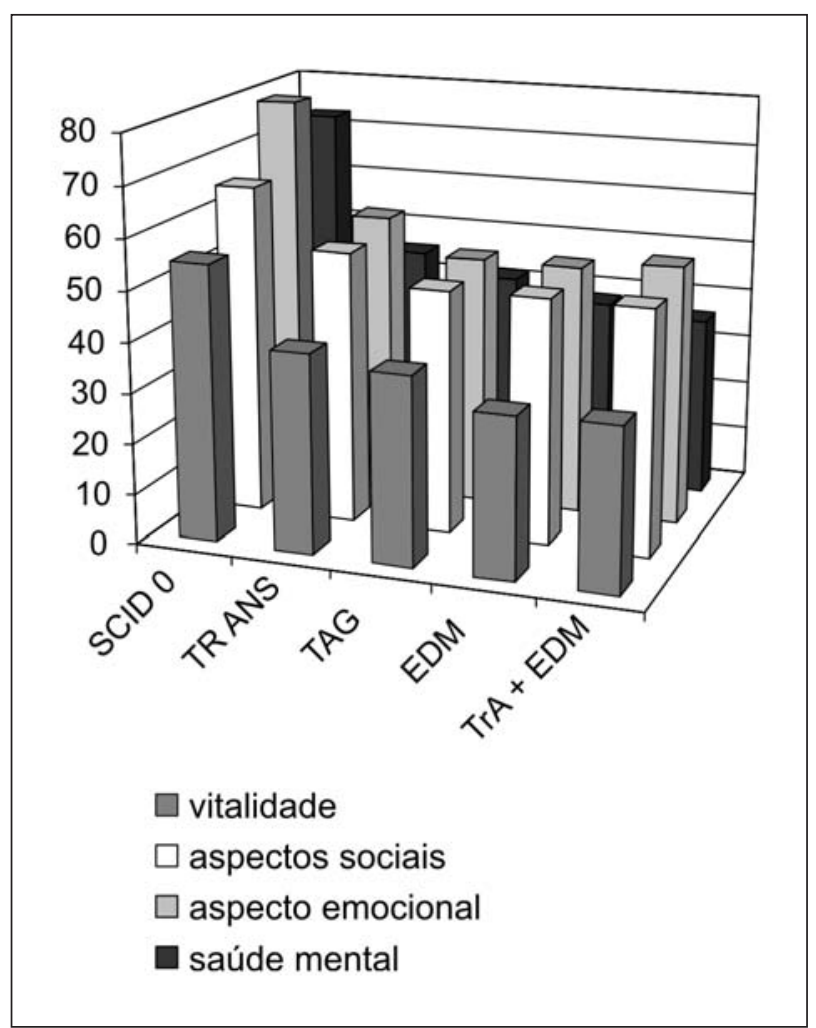

Fig 2. Distribuição dos escores da escala SF-36 nos subitens: vitalidade, aspectos sociais, aspecto emocional e saúde mental, em pacientes migranosos sem comorbidade psiquiátrica (SCID $0)$, com algum transtorno de ansiedade (Tr ANS), com transtorno de ansiedade generalizada (TAG), com algum episódio depressivo (EDM), e com transtorno de ansiedade mais episódio depressivo (TrA + EDM). 
ciada a comorbidade psiquiátrica tais como transtornos de ansiedade, transtorno de ansiedade generalizada, transtornos de ansiedade com pelo menos um episódio depressivo maior, assim como os pacientes apenas com episódio depressivo maior, comparados aos pacientes com enxaqueca crônica sem comorbidade psiquiátrica. $(p<0,05)$.

A Figura 1 mostra a distribuição dos escores da escala SF-36 nos aspectos físicos em pacientes portadores de enxaqueca crônica, sem comorbidade psiquiátrica (SCID 0), com algum transtorno de ansiedade, com transtorno de ansiedade generalizada, com algum episódio depressivo maior, e com transtorno de ansiedade mais episódio depressivo maior.

A Figura 2 mostra a distribuição dos escores da escala SF-36 nos aspectos mentais em pacientes portadores de enxaqueca crônica, com e sem comorbidade psiquiátrica (SCID 0). Observamos que os pacientes portadores de enxaqueca crônica sem comorbidade psiquiátrica apresentam melhor qualidade de vida nos aspectos físicos e mentais na distribuição dos escores da escala SF-36, comparados aos pacientes com comorbidade psiquiátrica. Os domínios dor e vitalidade apresentaram os escores de pior qualidade de vida.

Quando comparados os escores da escala SF-36 em pacientes com enxaqueca crônica com ausência de transtorno mental (SCID 0) e pacientes com enxaqueca crônica com comorbidade psiquiátrica, observamos que todos os domínios apresentaram diferença estatisticamente significante. Somente no domínio Estado Geral de Saúde do SF-36, a qualidade de vida não foi significativamente inferior em todos os grupos de pacientes com enxaqueca crônica associada a comorbidade psiquiátrica. Nos domínios do SF36 relacionados aos aspectos físicos, a qualidade de vida não foi significativamente inferior somente nos transtornos de ansiedade (Tabela 2).

\section{DISCUSSÃO}

Estudos relatam que indivíduos com enxaqueca apresentam escores de qualidade de vida inferiores aos indivíduos sem enxaqueca. Enxaqueca e depressão tem alta comorbidade e cada uma exerce significativa influência na qualidade de vida ${ }^{20,21}$. Porém, a literatura nacional e internacional não apresenta um estudo que avalie o impacto das comorbidades psiquiátricas na qualidade de vida de pacientes com enxaqueca crônica.

Nossos resultados mostram que a comorbidade da enxaqueca crônica com transtornos mentais é fator associado à piora da qualidade de vida dos pacientes com enxaqueca crônica. Todos os aspectos da escala SF-36 apresentaram diferença estatisticamente significante, com exceção do item estado geral da saúde para todos os grupos e aspectos físicos, quando comparados os pacientes com transtornos de ansiedade com os pacientes sem diagnóstico de transtorno mental. Como estamos analisando uma amostra de pacientes relativamente pequena, é possível que os resultados estejam influenciados por um erro de tipo beta, ou seja, deixamos de ver uma diferença que é real, porém o tamanho da amostra não permite poder estatístico suficiente para o detectarmos. Esta é a explicação mais plausível, já que todos os outros domínios mostraram diferença estatística significativa.

Um grupo controle sem cefaléia e um com enxaqueca episódica acrescentaria, na discussão, a possibilidade de avaliarmos se apenas o fator comorbidade psiquiátrica seria o responsável pela diminuição da qualidade de vida dos pacientes com enxaqueca crônica. Este pode ser um estudo preliminar a futuros trabalhos com este direcionamento.

Nosso estudo mostra e quantifica através dos diagnósticos psiquiátricos e da avaliação da escala de qualidade de vida nos pacientes com enxaqueca crônica que a presença das comorbidades de fato leva a redução na qualidade de vida destes pacientes, portanto implicações práticas devem ocorrer. A detecção de transtornos mentais em pacientes com enxaqueca mostra-se imprescindível para seu manejo mais adequado. A falha em reconhecer o diagnóstico e deixar de tratar tais condições pode levar a um desfecho clínico desfavorável, determinando para o paciente uma má qualidade de vida.

\section{REFERÊNCIAS}

1. Ciconelli RM. Medidas de avaliação de qualidade de vida. Rev Bras Reumatol 2003;43:9-13.

2. Subcomitê de Classificação das Cefaléias da Sociedade Internacional de Cefaléia. Classificação Internacional das Cefaléias. 2.Ed. Trad. Sociedade Brasileira de Cefaléia. São Paulo: Segmento Farma Editores, 2004:51-56

3. Castillo J, Munoz P, Guitera V, Pascual J. Epidemiology of chronic daily headache in the general population. Headache 1999;38:497-506.

4. Scher AI, Stewart WF, Liberman J, Lipton RB. Prevalence of frequent headache in a population sample. Headache 1998;38:497-506.

5. Lu SR, Fuh JL, Chen WT, Juang KD, Wang SJ. Chronic daily headache in Taipei, Taiwan: prevalence, follow-up and outcome predictors. Cephalalgia 2001;21:980-986.

6. Silberstein SD, Lipton RB, Solomon S, Mathew NT. Classification of daily and near-daily headaches: proposed revisions to the IHS criteria. Headache 1994;34:1-7.

7. Lipton RB, Stewart WF, Diamond S, Diamond ML, Reed M. Prevalence and burden of migraine in the United States: data from the American Migraine Study II. Headache 2001;41:646-657.

8. American Psychiatric Association. Diagnostic and statistical manual of mental disorders. 4.Ed. Washington: APA Press, 1994.

9. Regier DA, Myers JK, Kramer M, et al. The NIMH Epidemiologic Catchment Area program: historical context, major objectives, and study population characteristics. Arch Gen Psychiatry 1984;41:934-941. 
10. Kessler RC, McGonagle KA, Zhao S, et al. Lifetime and 12-month prevalence of DSM-III-R psychiatric disorders in the United States. Results from the national comorbidity survey. Arch Gen Psychiatry 1994;51:8-19.

11. Almeida-Filho N, Mari J, Coutinho E, et al. Brazilian multicentric study of psychiatric morbidity: methodological features and prevalence estimates. Br J Psychiatry 1997;171:524-529.

12. Goadsby PJ, Boes C. Chronic daily headache. J Neurol Neurosurg Psychiatry 2002;72(Suppl 2):S2-S5.

13. Merikangas KR, Angst J, Isler H: Migraine and Psychopathology: Results of the Zurich cohort study of young adults. Arch Gen Psychiatry 1990;47:849-853.

14. Breslau N, Schultz LR, Stewart WF, Lipton R, Welch KM. Headache types and panic disorder: directionality and specificity. Neurology 2001; 56:350-354.

15. Guillem E, Pelissolo A, Lepine JP. Mental disorders and migraine: epidemiologic studies Encephale 1999;25:436-442.

16. Merikangas KR, Merikangas JR, Angst J. Headache syndromes and psy- chiatric disorders: association and familial transmission. J Psychiatr Res 1993;27:197-210.

17. Guidetti V, Galli F, Fabrizi P, et al. Headache and psychiatric comorbidity: clinical aspects and outcome in an 8-year follow-up study. Cephalalgia 1998;18:455-462.

18. Ware JE, Sherbourne CD. The MOS 36-item Short-form Health Survey (SF-36). Medical Care 1992;30:473-483.

19. First MB, Spitzer RL, Gibbon M, Williams JBW. The structured clinical interview for DSM-IV axis I disorders: patient edition (SCID I/P, version 2.0). New York: Biometrics Research Department, New York State Psychiatric Institute, 1995.

20. Lipton RB, Hamelsky SW, Kolodner KB, Steiner TJ, Stewart WF. Migraine, quality of life, and depression. A population-based case-control study . Neurology 2000;55:629-635.

21. Terwindt GM, Ferrari MD, Tijhuis M, Groenen SMA, Picavet HSJ, Launer LJ. The impact of migraine on quality of life in the general population. Neurology 2000;55:624-629. 